\title{
Under construction: The geological GIS of the 1 : 5000000 International Geological Map of Europe and Adjacent Areas
}

\section{Introduction}

The 1:5 million International Geological Map of Europe and Adjacent Areas (IGME 5000) is a major European GIS project. It is being managed and implemented by the Federal Institute for Geosciences and Natural Resources (BGR) under the aegis of the CGMW (Commission of the Geological Map of the World). The project involves more than 48 European and adjacent countries and the final area covered will reach from the Caspian Sea in the east, to the Mid-Ocean Ridge in the west, and from Svalbard to the southern shore of the Mediterranean Sea (Figure 1). The aims of the project are to develop a GIS underpinned by a geological database that will deliver a printed map providing up-to-date and consistent geological information.

The GIS will hold significantly more information than the first paper edition of the map (Bundesanstalt für Bodenforschung and UNESCO, 1971) could ever provide. It will also offer versatility, e.g. to retrieve and present for the whole of Europe, information on age, petrography, structural and metamorphic features. More importantly the IGME 5000 GIS will provide the essential foundation for pan-European applied geo-environmental thematic mapping. While the main theme of the GIS is the pre-Quaternary geology of both the land and offshore areas of Europe, it is planned to include additional themes, such as Quaternary geology: a key factor influencing the natural landscape. In addition to the printed map, it is planned to release an internet version of the GIS allowing the retrieval and downloading of map components in graphic and table form. It is already possible to download the preliminary map legend, the topographic base map and other information like project news from the IGME 5000 website (www.bgr.de/karten/IGME5000/). A CD-ROM will also be produced with a subset of the GIS and the related database. An extensive multinational project like the IGME 5000 requires meticulous preparation and establishment of standards and protocols in order to provide the essential structure and guidelines for the data compilation e.g. common term dictionaries for the database. In addition, a standard topographic base map was an essential prerequisite. So in many areas the IGME 5000 is establishing basic standards where none exist.

\section{Aims and objectives}

The overall aim of the IGME 5000 project is to prepare a geological GIS (Geological Information System) for Europe, allowing the flexible retrieval of geological attributes and the production of a printed geological map. The full project objectives are to:

\section{1:5 Million International Geological Map of Europe and Adjacent Areas}

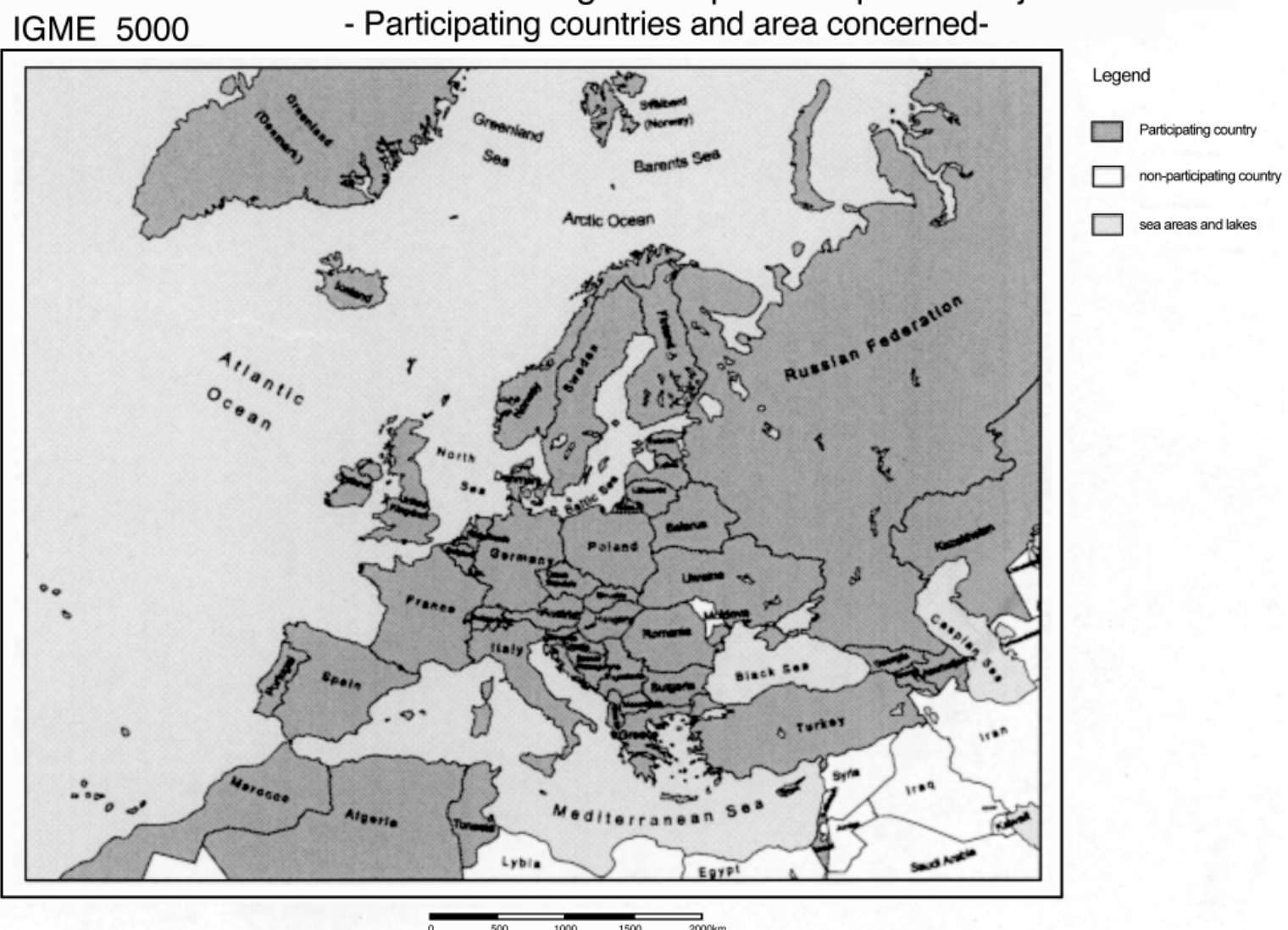

Figure 1 The IGME 5000 project area and participants. 
- display the current status of Europe's geology,

- display the off-shore geology for the first time (and ensure its consistency with on-shore),

- clarify the correlation of European geology, both on-shore and off-shore,

- establish and intensify co-operation between and with national geological institutions,

- encourage new research subjects,

- provide with Europe-wide usable guidelines and procedures,

- establish simple European geological standards such as term dictionaries and colour schemes,

-develop a robust and executable model for comparable projects,

- produce user-tailored thematic maps,

- allow retrieval and combination of diverse geological attributes,

—and last but not least to make all this information available via the Internet.

\section{Co-operation with international participants and scientific advisors}

Because the project involves so many European and adjacent countries, mobilising the respective national geological surveys was essential. They were required to contribute draft maps of the preQuaternary geology of their on- and off-shore areas and therefore required consistent technical guidance on procedures and the provision of standards. A project like the IGME 5000 is totally dependent on the cooperation of the participants. The fact that so many geologists are prepared to willingly make a voluntary contribution is indicative of their dedication to their science (Asch and Jackson, 2001).

Four years on a growing number (latest count: 48) of Geological Surveys of Europe, North Africa and the Middle East are involved in the IGME 5000. A network of scientific advisors has been built up; these people are either providing individual advice or are consulted in workshops on special issues, such as the marine or Alpine geology. The IGME 5000 advisers are mainly academic experts and/or members of international geoscience institutions.

\section{Development of common standards, guidelines and protocols}

\section{The need for common standards}

Organising the co-operation of the numerous participating nations and compiling their input is a considerable information management task. This comprises data acquisition, meaning in this context motivating the numerous participants to contribute appropriately. It involves subsequent data harmonisation and generalisation and thus coping with different interpretations, a variety of approaches to generalisation and drawing quality from 48 individual national geological institutions, as they establish what they believe should be displayed of their geology on a Europe-wide map. The ultimate goal is the unification of all data to build a harmonious whole of these individual pieces of the European geological jigsaw puzzle.

Given the idiosyncratic character of geologists each having their own interpretation of the geological environment and the development of different geological classification systems, discrepancies in the following were not unexpected:

- geological attributes, such as lithological and chronostratigraphical descriptors,

—mapped units (emphasis, number, ...), -topographic base (co-ordinate system, ellipsoid, drainage system, projection),

- draft map scale,

-level of detail,

- colours, symbols,

— data structures and hierarchies.

Thus, consistent procedures (and workflows) needed to be established at the outset that nevertheless allowed the addition of countries (and requirements) joining later. In connection with this, appropriate standards, protocols and guidelines were developed to ensure that those contributing to the IGME 5000 were working on the same geological, cartographical and geographical (topographical) basis and therefore supplied consistent data.

\section{The "Guidelines": Preliminary legend and technical guidance}

In order that the geological draft map could be created by each participant for the area of their country, a preliminary map legend (containing a provisional classification of the geological units) was developed. This legend defines the contents of the map (and at the same time places necessary constraints on the contributing geologists). A number of partly hierarchically organised tables of age, petrography and metamorphism of the rock units make up this legend. More basic, but nonetheless critical, was the distribution of simple technical guidance notes for the preparation of the draft maps. Guidelines were designed (Asch, 1997) which introduced a scheme for the draft map preparation, defined specifications which the submitted draft should meet, advised upon the level of generalisation and additionally contained the preliminary legend described above. The guidelines may also be downloaded on the IGME 5000 web site.

\section{Common topographic base map}

Of fundamental importance is a common topographic base map. For the IGME 5000 project a common topographic base map not only controls the spatial referencing of the data but also needs to be suitable for printing. The map is now available digitally for all participants for free on the IGME 5000 website. All IGME 5000 contributors were asked to use this common topographic base map and to submit their contributions on it, identifying their geological units using an individually created, unique identification number which would be related to the general legend. The submission of the draft maps was and is preferred in analogue form to avoid potential software incompatibilities and also to avoid the need for digitising at the partner institutions.

\section{Colour schemes and abbreviation codes}

To display geological features on a map, in Europe, conventionally a colour scheme is used that is based on the colours of the spectrum for sedimentary rocks (see the IUGS stratigraphic chart, 2000). Also red tones are used conventionally for igneous rocks. The IGME 5000 follows this convention so as to make the map readable to as many users as possible, even if they may not understand the language (English) of the map. However, in other continents such as North America or Australia, different colour scheme conventions are in use. To facilitate access for those users not familiar with the European convention, such options will be provided; this is possible because the IGME 5000 is digitally based. The GIS will also allow the display of the rock units according to a lithological or stratigraphically oriented colour scheme.

\section{Term dictionary for a $1: 5000000$ scale}

An essential part of any GIS is a well-designed database and thus definition of its content and structure are key tasks. The term dictionaries were compiled on the basis of existing international standards as far as possible (e.g. for the classification of igneous rocks from 
Streckeisen, 1973, and 1980; the chronostratigraphic classification of sedimentary rocks from the IUGS, 1998.) Approximately 300 geological terms are listed so far, however, until the map is finished discussion on the definition of these terms will continue.

\section{Data structure and data input mask}

To facilitate data acquisition and to involve the participating institutions in the population of the database, a data input screen (or template, see Figure 2) using a MS Access runtine version was distributed to the IGME 5000 participants. Based on the term dictionaries and a comparatively simple data model, the screen provides information and limits on how the geological units may be described. Although simple, the data model had to be developed with the potential for later modification to a more sophisticated version and moreover it had to facilitate the necessary synthesis of the individual "country databases".

The descriptions provided are being collected in "country databases" at the participating geological institutions. When the data acquisition is complete, the mask system also includes functions to compress and copy the individual database for mailing or e-mailing back to BGR (and to create a back-up for local use).

\section{Data harmonisation and synthesis}

The project is currently at the stage of data harmonisation and synthesis.

\section{Draft maps}

After the national draft maps are sent back to the BGR (and despite all the pre-emptive efforts to ensure consistency), the geological units and even the individual legends (because of the enormous variety of geological units) are being cartographically and geologically harmonised and generalised. Figure 3 illustrates some of the issues which arise.

\section{Database and GIS}

To create the database the following process is employed: on integrating the country-related data tables, the definite identification numbers (IN, see above) are harmonised by applying an algorithm so that ultimately only one combination of attributes relates to one identification number. This newly generated IN replaces the original IN national numerical coding of the geological units. The BGR project team manually allocates the new IN to the units on the draft maps in

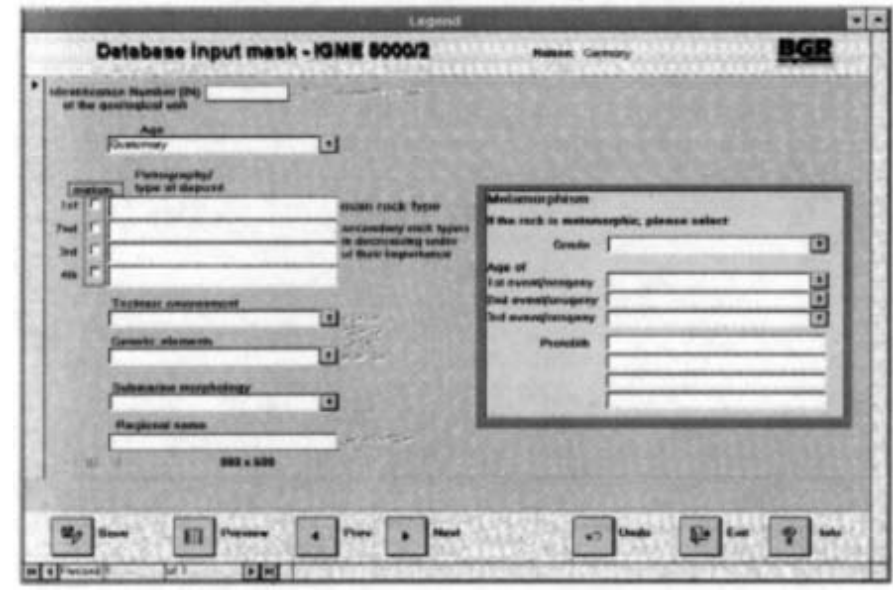

Figure 2 The IGME 5000 data input screen, distributed to all project participants.

the course of the geological harmonisation and the geological and cartographical generalisation.

After collation the linework of the European geological map will be digitised and transferred to and further processed in ArcInfo, the GIS platform on which the the IGME 5000 is currently being developed. The primary key to the descriptions in the database will be provided by the IN after they have been added as attributes to the geological polygons. The entire procedure finally delivers a database that comprises the attributes of all geological units and their IN as a link to a complete and harmonious European geological map with a unique IN for all areas.

\section{Current status and future progress/plans}

To date the IGME 5000 project has achieved the following milestones:

- The digital topographic base map is available to all participants and associated projects on the BGR web server.

- The term dictionaries ("thesaurus"), still in the process of optimisation, are implemented as standard for the database.

- The data input mask ("template") has been programmed and distributed.

- The "proto" design and structure of the geological database is complete.

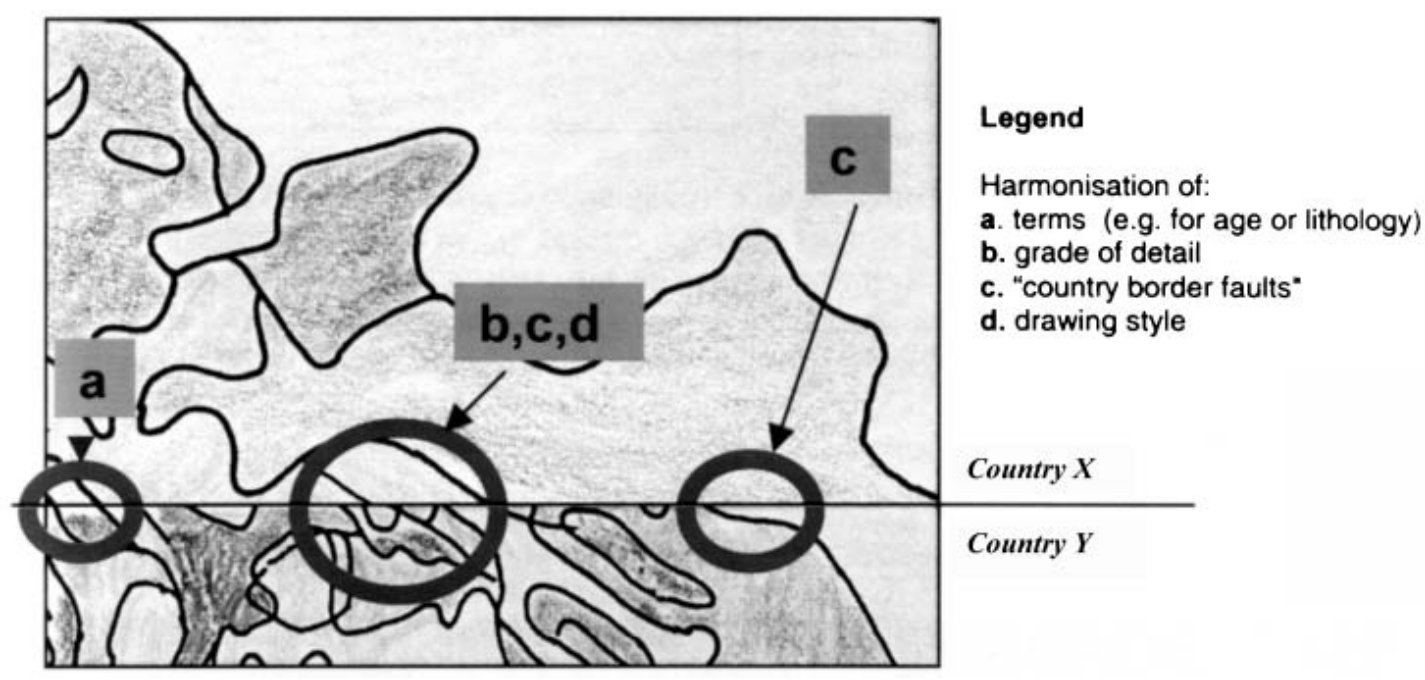

Figure 3 Harmonisation of the various draft maps of the countries. 
- A colour legend and abbreviation code has been developed and is available on the IGME 5000 web site.

-Advisory groups on marine and alpine geology are established and active.

- The geological GIS and draft map are in the process of construction.

- Cooperation with other European projects such as GEIXS, GESTCO and the Oil and Gas Atlas is established.

It is hoped that the map will be printed in 2002, with the complete web version being released later that year. The GIS that will result from the project will not only enable the production of a printed map, but also an interactive CD-ROM, an internet version and extract maps to be produced on demand. More significantly perhaps it will provide a much-needed foundation for further pan-European research, standardisation and geoscientific and environmental thematic mapping.

\section{Acknowledgements}

The author wishes to acknowledge the invaluable contribution to the project of all the national participants and the IGME 5000 team members in the BGR. Without their commitment and hard work the project would not have progressed so far and this paper could not have been written. I would also like to record my thanks to other colleagues in the BGR for help and advice during this project and especially to Prof. Dr. F.-W. Wellmer for his continuing support.

\section{References}

Asch, K., 1997, Guidelines for the Preparation of Draft Maps; Federal Institute for Geosciences and Natural Resources, Hannover.

Asch and Jackson, 2001, No Pain No Gain: The reality of initiating and managing three pan-European Geoscience Information System projects, Episodes, v. 24, no. 1, pp. 32-36.

BGR and UNESCO, 1971, International Geological Map of Europe and the Mediterranean Region, 1 : 5 Million; Hannover.

IUGS, 2000, International Stratigraphic Chart, Strasbourg.

Streckeisen, A.L., 1973, Classification and Nomenclature recommended by the IUGS Subcommission on the Systematics of Igneous Rocks. Geotimes, 10/73, pp. 26-31

Streckeisen, A.L., 1980, Classification and Nomenclature of Volcanic Rocks, Lamprophyres, Carbonites and Melilitic Rocks. IUGS Subcommission on the Systematics of Igneous Rocks. Band 69, v. 1; pp. 194-207.

\section{Kristine Asch}

Federal Institute for Geosciences and Natural Resources (BGR) Stilleweg 2, D-30655 Hannover GERMANY

Phone: ++495116433324 ,

Fax: ++495116433782

E-mail: kristine.asch@bgr.de

Internet: www.bgr.de/karten/IGME5000

\section{Hutchison 'Young Scientist' Fund}

William Watt Hutchison, "Hutch" to his many friends around the world, was a Scots-born Canadian geologist who served Canada and the IUGS in myriad dynamic and creative ways. Most notably, he served as the IUGS Secretary General (1976-1980) at a pivotal time in its history, and as IUGS President (1984-1987). The same boundless energy, enthusiasm, skill in communications, and ability to foster teamwork that characterized his work with the IUGS also carried him to preeminent scientific administrative positions in the Canadian Government, where he served as Director General of the Geological Survey of Canada and as Assistant Deputy Minister of Earth Sciences. His distinguished career was terminated in 1987 by his untimely death at the age of 52, following a painful struggle with cancer.

One of Hutch's last wishes was to establish under IUGS auspices a memorial foundation intended to promote the professional growth of deserving, meritorious young scientists from around the world by supporting their participation in important IUGS-sponsored conferences. The first 3 beneficiaries of the Hutchinson "Young Scientist Foundation" attended the 28th International Geological Congress (IGC) in Washington, D.C., in 1989. In 2000, 6 awardees received financial support from the Hutchison Fund for attending the 31st IGC in Rio de Janeiro, Brazil.

The Hutchison "Young Scientist Foundation" is a worthy cause that honors a fine, caring man and a distinguished, public-spirited scientist and administrator. The foundation also celebrates and promotes those things that gave Hutch the most professional satisfaction: geology, international scientific collaboration, and stimulating young minds.

The IUGS welcomes contributions to the Hutchison "Young Scientist Foundation." Please send donations to:

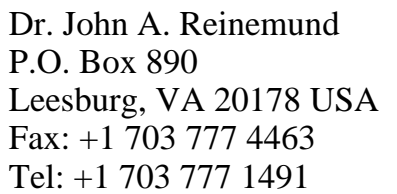

Checks in US dollars or Visa/Mastercard (please include account number and expiration date) are preferred in order to avoid the high cost of currency conversions. Residents of the U.S.A. are reminded that charitable gifts of this nature are tax deductible. 